\title{
GÉNERO Y CURRICULUM: UNA MIRADA GENERIZADA SOBRE EL PLAN DE ESTUDIOS DE LA CARRERA DE SOCIOLOGÍA
}

\author{
GENDER AND CURRICULUM: A GENDER PERSPECTIVE ON THE SOCIOLOGY DEGREE \\ CURRICULUM
}

https://orcid.org/0000-0001-7612-5449 Andres Malizia ${ }^{A}$

${ }^{\text {A }}$ Universidad de Buenos Aires (UBA), Buenos Aires, Argentina

Recebido em: 30 abr. 2021 | Aceito em: 29 ag. 2021 Correspondência: Andres malizia (camalizia@gmail.com)

\begin{abstract}
Resumo
En este artículo me propongo describir y analizar el plan de estudios de la carrera de Sociología de la Universidad de Buenos Aires, Argentina. Especialmente me interesa indagar sobre las formas en que aparecen explicitadas las problemáticas y perspectivas de géneros en los programas que conforman el ciclo de la licenciatura. Teniendo en consideración que las universidades son formadoras de docentes, en este trabajo analizo las características que asumen dichas perspectivas en el plan de estudios, entendiendo que el mismo nos ofrece una version posible acerca de cómo se forman en la universidad quienes pueden ejercer la docencia en la educación secundaria y superior. La intención final es la de producir una aproximación crítica sobre la formación universitaria en géneros y sexualidades.
\end{abstract}

Palavras-chave: formación docente; educación sexual; universidad.

\begin{abstract}
In this article I propose to describe and analyze the study plan for the Sociology major at the University of Buenos Aires, Argentina. I especially want to inquire the ways in which gender issues and perspectives (sex education) appear explicit in the programs that make up the undergraduate cycle. Bearing in mind that universities are teacher educators, in this paper I analyze the characteristics that sexuality education assumes in the curriculum, understanding that it offers us a possible version of how those who can teach at university are trained in secondary and higher education. The final intention is to produce a critical approach to university education in genders and sexualities.
\end{abstract}

Keywords: teacher training; sex education; university. 


\section{Educación sexual, universidad y autonomía}

La Ley Nacional de Educación Sexual Integral (ESI) sancionada a finales del 2006 se enlaza a una tendencia a nivel regional influenciada por las directrices de organismos internacionales (BAEZ, 2016), y en confluencia con las demandas del movimiento de mujeres y de los movimientos socio-sexuales de la Argentina (FELITTI, 2009). La larga trayectoria de estas organizaciones, junto con un sector de la sociedad que adhería y la voluntad política que acompañaba en las distintas cámaras del Poder Legislativo, hicieron posible la sanción de la ley de ESI, y de otras leyes vinculadas al campo de géneros y sexualidades. Como afirma Barrancos (2014) las problemáticas de género datan -en muchos casos- desde mediados del siglo XX, pero a partir de la vuelta a la democracia en 1983, se fueron consolidando las agrupaciones y organizaciones de mujeres, feministas y de la disidencia socio-sexual que, teniendo diferencias entre sí, lograron encausar ciertas políticas de unidad que las llevaron a transitar reclamos comunes y a obtener logros de enorme significado.

Distintxs investigadorxs ${ }^{\mathrm{i}}$ (PETRACCI y RAMOS, 2006; PECHENY y PETRACCI, 2006) coinciden al afirmar que la restauración de la democracia generó en la Argentina una etapa de transición, marcada por el pasaje de un periodo de restricciones y omisiones a otro de deliberación y movilización política. En ese periodo los reclamos por los derechos humanos fueron adquiriendo mayor visibilidad y configurando la agenda política (PETRACCI y RAMOS, 2006). En sintonía, Pecheny y Petracci (2006) plantean que la "vuelta a la democracia" trajo consigo el surgimiento de nuevos actores sociales y dio apertura y amplitud al universo político: los temas de la agenda política comenzaron a cuestionarse y a deliberarse públicamente. En ese contexto, de reivindicación de los derechos humanos, se politizaron las relaciones sociales, se cuestionaron los límites entre lo público y lo privado, entre lo natural y lo social. La politización pasó entonces por demostrar que las relaciones consideradas privadas están en realidad atravesadas por una dimensión política y que las relaciones percibidas como naturales son en realidad construidas social e históricamente (PECHENY y PETRACCI, 2006).

En ese escenario, nuevos espacios se volvieron terreno fértil para las acciones políticas, las movilizaciones y las exigencias hacia el Estado. Los debates públicos y parlamentarios, junto con las acciones llevadas adelante por el movimiento de mujeres, fueron generando las condiciones necesarias para la sanción de nuevas leyes vinculadas con la sexualidad como la ley de Salud Sexual y Procreación Responsable ( ${ }^{\circ}$ 25.673/03) y la ley de Educación Sexual 
Integral ( $\mathrm{N}^{\circ}$ 26.150/06). En ese proceso, también se incorporaron a la Constitución Nacional algunos tratados y acuerdos internacionales como la Convención sobre la Eliminación de todas las formas de Discriminación contra la Mujer (CEDAW, por su sigla en inglés) incorporada en la reforma constitucional de 1994.

La inclusión de la dimensión de género en la política educativa fue un proceso complejo de disputas, alianzas y negociaciones entre los distintos actores sociales (LAVIGNE,2016; MORGADE et al 2016). En el año 2006 se sancionó la ley 26.150 de creación del Programa Nacional de Educación Sexual Integral (ESI). La misma aclara que "todos los educandos tienen derecho a recibir educación sexual integral en los establecimientos educativos públicos, de gestión estatal y privada de las jurisdicciones nacional, provincial, de la Ciudad Autónoma de Buenos Aires y municipal” (artículo 1º), y que "las acciones están destinadas a quienes asisten a establecimientos educativos desde el nivel inicial hasta el nivel superior de formación docente y de educación técnica no universitaria" (artículo $4^{\circ}$ ).

Desde su sanción, los distintos niveles del sistema educativo se han visto interpelados por la ESI. Principalmente porque instaló un cambio en la concepción con respecto a la sexualidad, comprendiéndola desde la integralidad (como la articulación de los aspectos biológicos, psicológico, sociales, afectivos y éticos) y no desde una mirada biomédica y moralizante $^{\mathrm{ii}}$ (MORGADE y ALONSO, 2008). Además estableció que la educación sexual es un derecho y una obligación de las instituciones educativas, y que la misma debe enseñarse teniendo en cuenta 5 ejes: el reconocimiento de la perspectiva de género, la valoración de la afectividad, el cuidado del cuerpo y de la salud, el respeto por la diversidad y el ejercicio de los derechos.

Estas características y dimensiones adjudicadas y apropiadas por la ESI, llevaron a que el sistema educativo, desde la educación inicial hasta la educación superior-formación docente, se viera interpelado durante los últimos catorce años. Si bien la letra de la ley no contempla específicamente a las universidades debido a la autonomía universitaria, desde distintas investigaciones (MORGADE et al, 2018; MORGADE y FAINSOD, 2015) se viene cuestionando su lugar y responsabilidad social. Si las universidades son instituciones educativas y formadoras de docentes, ¿pueden quedar al margen de la ESI? ¿qué responsabilidad tienen en la formación docente? Si lxs estudiantes universitarixs son educandxs (tal como se plantea en la ley) y si la educación sexual integral es un derecho, ¿de qué forma, en las aulas y en las 
instituciones universitarias, se garantiza? ¿cómo permea la ESI a las aulas universitarias? ¿qué ocurre en ellas? ¿la autonomía universitaria puede impedir su abordaje?

Cuando estas inquietudes se sitúan en el terreno universitario se renuevan las preguntas acerca de los idearios, las expectativas e imaginarios sobre la institución y la formación universitaria (BAEZ, 2020). ¿Hasta qué punto la universidad se reconoce como formadora en educación sexual y como formadora de docentes? Ante estas preguntas, algunas investigaciones como las de Blanco (2014) apuntan a desentrañar el carácter sexuado de la vida universitaria, incursionando en el análisis de los modos de construcción identitaria a través de las regulaciones sexo-genéricas al interior de la universidad. En tanto que otras investigaciones (SARDI, 2017; MORGADE, 2017) se centran en la problematización de la construcción de los conocimientos científicos y de la formación disciplinar de las carreras, reconociendo el carácter androcéntrico y sexista de las mismas.

En consonancia con las últimas investigaciones mencionadas, en este artículo comparto los análisis sobre el plan de estudios de la carrera de sociología, que contempla el relevamiento de 37 programas correspondientes al bloque obligatorio de la licenciatura. De este modo, este trabajo de indagación permite comprender los modos en que las problemáticas de géneros y sexualidades y la educación sexual, han permeado el curriculum de grado, y posibilita, a su vez, una aproximación crítica sobre la formación de profesorxs para la educación secundaria y superior en sociología.

Este trabajo se inscribe en el proyecto de investigación que estoy llevando adelante, "Género y formación docente: sujetos, saberes y experiencias" y en el trabajo de investigación del equipo del cual formo parte, "Formación docente, androcentrismo científico y educación sexual integral con enfoque de género", ambos radicados en el Instituto de Investigaciones en Ciencias de la Educación de la Facultad de Filosofía y Letras de la Universidad de Buenos Aires (IICE-FFyL-UBA).

Antes de iniciar, cabe mencionar que el hecho de analizar el plan de estudios y los programas que conforman esta carrera plantea limitaciones y potencialidades. Por un lado, permite adentrarse a la carrera reconociendo las asignaturas que incorporan estas problemáticas y perspectivas, y por otro, al acercarse desde lo prescriptivo del plan, queda por fuera lo que ocurre en las aulas, lo que podemos denominar como curriculumexplícito (SACRISTAN, 1997), oculto (JACKSON, 1968) o nulo (EISNER, 1985). Aunque este abordaje presente sus limitaciones, su relevamiento es necesario y valioso, ya que permite inferir acerca de la importancia que presentan estas temáticas en la carrera y en las asignaturas que lo abordan. 
Como así también, al tratarse de una elaboración que es producto de discusiones, debates y negociaciones (DE ALBA, 1995), el análisis de los programas y del plan de estudios permite vislumbrar el carácter político que envuelve a todo proceso educativo.

\section{Sociología y educación sexual, un recorrido por los planes de estudios}

La carrera de Sociología se creó en el ámbito de la Universidad de Buenos Aires en el año 1958. A lo largo de los sesenta años de existencia se han aprobado cinco planes de estudio. El primer plan data de 1958, el segundo de 1974, el tercero de 1976, el cuarto de 1985 y el quinto -y actualmente vigente- de $1988^{\text {iii }}$.

Como plantea Mancuso (s/f) cada uno de estos planes se ancla en diferentes contextos históricos del país y de la Universidad. Los primeros dos planes tenían una impronta marcada por la metodología de la investigación y las estadísticas. Se ofrecía una amplia variedad de

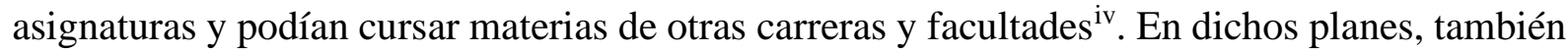
se dejaba entrever una preocupación por la articulación entre teoría y práctica. Por ese motivo, se incorporó -desde el primer plan- la participación en trabajos de investigación, pudiendo ser en cátedras o institutos de investigación. A partir del segundo plan, se establece una diferenciación entre "teorías sociológicas" y "sociologías especiales", posibilitando el acercamiento a las ramas de la sociología y sus especialidades. Ambas características: participación en equipos de investigación (por un lapso de 200 hs.) y distinción entre teorías y sociologías especiales perduran hasta la actualidad.

Con la dictadura cívico-militar de 1976 se ve afectada la vida institucional de la Universidad (GOGNA et al. 2010). En lo que respecta a la carrera, con la reforma del plan de estudios de 1976 se desarmó el pluralismo de escuelas sociológicas, quitando la posibilidad de cursar asignaturas y reduciendo la metodología de la investigación a meras técnicas estadísticas (MANCUSO, s/f). El modelo reinante era acorde al cerramiento ideológico que marcaba en ese entonces a todo el sistema educativo de la Argentina. Con la llegada de la democracia en 1983, se genera un proceso de normalización de las instituciones que implicó la intervención de la Universidad, la creación de Consejos y la puesta en vigencia de Estatutos previos a 1976. En ese contexto, de recuperación de la vida democrática, se aprueba en 1985 el cuarto plan de estudios, que proponía -a través de materias obligatorias y específicas-, instaurar nuevamente la especificidad sociológica. Este plan estuvo vigente por pocos años, ya que en 1988 se aprobó el quinto plan. Siguiendo los aportes de Mancuso, el plan de 1985 no perduró porque estaba 
deslegitimado institucionalmente, ya que, los representantes del claustro de estudiantes lo habían impugnado y en el marco de la reconstrucción de la carrera, está condición no daba cuenta del perfil pluralista que se pretendía.

En relación a la formación de profesorxs para la educación secundaria y superior, en los planes de 1958, 1974 y 1976, dicha titulación se encontraba integrada al mismo ciclo de la licenciatura, es decir, que la propia organización curricular contemplaba en los últimos años la cursada de 4 asignaturas: Didáctica General, Psicología de la Niñez y la Adolescencia, Pedagogía y Práctica de la Enseñanza. De esta forma, al finalizar la carrera se podía egresar con ambos títulos: licenciatura y profesorado. En el plan de 1985 se tenían que aprobar 3 asignaturas: Didáctica, Pedagogía y Práctica de la Enseñanza, además de realizar una pasantía anual en instituciones públicas o privadas.

El plan de 1988 establece que la licenciatura está conformada por 31 asignaturas: 6 corresponden al Ciclo Básico Común $(\mathrm{CBC})^{\mathrm{v}}, 16$ a las obligatorias y 9 a las optativas ${ }^{\mathrm{vi}}$. Además, tienen que acreditar 200 horas de investigación o cursar seminarios y/o talleres anuales o cuatrimestrales y aprobar 3 niveles de idioma a elección (inglés o francés). El profesorado se constituye en un ciclo propio, conformado por 3 asignaturas: Didáctica, Pedagogía y Práctica de la Enseñanza, y se determina que para acceder a dicho ciclo es necesario contar previamente con el título de la licenciatura, es decir, que a partir de 1988 solo pueden cursar el profesorado quienes sean licenciadxs en sociología.

En este artículo se aborda como objeto de análisis el bloque conformado por las asignaturas obligatorias del ciclo de la licenciatura. Teniendo en cuenta que las 16 asignaturas presentan cátedras paralelas, oscilando entre 2 y 3 cátedras por asignatura, el total de asignaturas y programas analizados asciende a 37. Las materias obligatorias se encuentran organizadas en cuatro áreas: teoría sociológica, historia social, metodológica y complementaria u "otras obligatorias" (como se menciona en el plan).

En la tabla que está a continuación se pueden observar las áreas, las asignaturas, la carga horaria y la cantidad de cátedras que tiene cada materia.

Tabla 1

\begin{tabular}{l|l|l}
\hline Área & Asignaturas y carga horaria & Cátedra \\
\hline Teoría sociológica & -Sociología general $(84 \mathrm{hs})$. & -Dos \\
& -Sociología sistemática $(84 \mathrm{hs})$. & -Dos \\
& -Sociología política $(56 \mathrm{hs})$. & -Dos \\
& -Historia del conocimiento sociológico I (84 hs.) & -Dos \\
& -Historia del conocimiento sociológico II (84 hs.) & -Dos \\
\hline Historia Social & -Historia social moderna y contemporánea $(84 \mathrm{hs.})$ & -Dos \\
& -Historia social latinoamericana $(84 \mathrm{hs})$. & -Dos \\
\hline
\end{tabular}




\begin{tabular}{l|l|l}
\hline & -Historia social argentina (84 hs.) & -Dos \\
\hline Metodológica & -Epistemología de las ciencias sociales (84 hs.) & -Dos \\
& -Metodología I (84 hs.) & -Tres \\
& -Metodología II (56 hs.) & -Tres \\
& -Metodología III (56 hs.) & -Tres \\
\hline Otras obligatorias/ & -Filosofía (56 hs.) & -Dos \\
Complementaria & -Economía II (56 hs.) & -Tres \\
& -Análisis de la sociedad argentina (56 hs.) & -Dos \\
& -Psicología social (56 hs.) & -Tres \\
\hline
\end{tabular}

Fuente: elaboración propia en base al plan de estudios de 1988.

En esta primera etapa se analizaron las 37 asignaturas y programas indagando en cada una de ellas sobre la presencia de las problemáticas de géneros y sexualidades y de la perspectiva de género. En un primer momento del análisis, se realizó una búsqueda de palabras claves: "perspectiva de género", "desigualdades de género", "mujer/es", "feminismo", "disidencia/diversidad sexual", “erotismo", “placer", "sexualidad", “cuerpo", “machismo", "patriarcado", "violencia",entre otros. Luego se realizó una lectura comprensiva de la fundamentación, los objetivos, los contenidos y la bibliografía obligatoria, complementaria o sugerida. Y en un tercer momento, se contabilizó la bibliografía obligatoria y optativa, distinguiendo la identidad sexo-genérica de lxsautorxs.

Del análisis de los programas se pueden destacar tres aspectos: 1) el lugar que ocupan las problemáticas y perspectivas de género en el marco del bloque de asignaturas obligatorias; 2) los temas que se abordan en las materias y la forma en que se organizan curricularmente al interior de cada una; y 3) el canon de lectura que se ofrece a lxs estudiantes.

\section{Asignaturas obligatorias y educación sexual, ¿la ESI es obligatoria?}

$\mathrm{Al}$ analizar las 37 asignaturas que conforman el bloque obligatorio de la carrera, encontré que en 6 de ellas se explicitan estas problemáticas y perspectivas. Las mismas corresponden a Historia Moderna y Contemporánea, Historia Social Latinoamericana, Historia Social Argentina, Psicología Social y Epistemología de las Ciencias Sociales ${ }^{\text {vii }}$.

Este primer hallazgo comienza a generar algunos interrogantes en relación a las áreas en las que se organiza el bloque de asignaturas obligatorias. Las 6 asignaturas que incorporan estas problemáticas corresponden en mayor medida al área "historia social" y en menor medida al área "metodológica" y "complementaria". Hay dos aspectos que resultan llamativos en este acercamiento: en primer lugar, que en el área "teoría sociológica" -que se encuentra conformado por las asignaturas específicas de la disciplina (saberes sociológicos) y que a su vez presenta la mayor carga horaria-, no se mencionen explícitamente contenidos vinculados a estas 
problemáticas; y en segundo lugar, que las asignaturas que sí incorporan estas temáticas en sus programas aportan sus saberes de género desde otras disciplinas como historia, psicología y epistemología.

\section{¿Qué enseñan quienes sí incluyen estas problemáticas y perspectivas?}

El segundo aspecto a destacar refiere a los temas que se describen en los programas, como así también a las formas en que se organizan curricularmente al interior de cada uno de ellos.

En Historia Social Moderna y Contemporánea se plantea desde los objetivos de la asignatura la construcción de un enfoque interdisciplinario, entre la Sociología y la Historia, que contemple la multidimensionalidad de la realidad social. En este marco, las cuestiones de género se hacen presentes a través del análisis sobre el lugar que ocuparon las mujeres en distintos periodos históricos como la Revolución Francesa, la consolidación de los Estados nacionales, la organización del movimiento obrero y la República popular China: mujeres y ciudadanía; mujeres obreras y trabajo; mujeres y socialismo. También hay una referencia a los años sesenta, los movimientos sociales y los nuevos actores que surgieron como los jóvenes y la lucha de las llamadas "minorías".

En Historia Social Latinoamericana se propone el ejercicio de pensar los procesos sociales, políticos, económicos y culturales de la región durante el siglo XX. A partir del abordaje de 5 casos: México, Brasil, Cuba, Bolivia y Chile, se pretende problematizar los procesos sociopolíticos más relevantes: revoluciones, socialismo, populismo, dictaduras, entre otros, como así también conocer y reflexionar en torno a los sujetos sociales y sus agencias sin olvidar una impronta de género que nos parece importante desinvisibilizar. En los apartados dedicados a cada uno de estos casos se hace referencia a las cuestiones de género. Principalmente las menciones son sobre la participación de las mujeres: la evolución del feminismo y su participación en la oposición al régimen dictatorial; la condición de las mujeres y los movimientos reivindicativos.

En Historia Social Argentina se proponen como objetivo de la asignatura, realizar una historia social de la política y una historia política de lo social, cercana a una historia social del poder.La lectura histórica tendrá en cuenta el poder económico, mediático, financiero, militar, religioso, simbólico y patriarcal, evitándose todo tipo de reduccionismos, esencialismos, determinismos y miradas binarias de la sociedad. En los contenidos de la 
asignatura, se menciona explícitamente lo vinculado al voto: por un lado, los años 30 y el voto masculino obligatorio; y por otro, los años cuarenta y el voto femenino obligatorio.

En Psicología Social (cátedra A) se plantea un recorrido por un conjunto de núcleos temáticos, que incluyen dimensiones, nociones y problemas sobre la subjetividad y subjetivación, de importancia central en ciencias sociales contemporáneas. En la fundamentación del programa, el enfoque de género es presentado como una de las dimensiones que posibilitan la crítica a la matriz heterosexual, al dispositivo de la sexualidad y la indagación en los vínculos entre corporalidades y nuevas tecnologías. La asignatura se organiza en seis unidades: "Sujeto, persona y sí mismo", "Cuerpo", "Género", "Emociones”, "Tecnología, psiquismo y capitalismo" y "Subjetividades post-decoloniales". A lo largo de estas unidades se recorren los nuevos modos de producir sujetos, cuerpos, géneros, emociones y tecnologías, desde el capitalismo contemporáneo y las agendas post-decoloniales.

En Psicología Social (cátedra B) se define que la materia se posiciona con una mirada epistemológica en torno a distintas corrientes de estudio del fenómeno psicosocial, en el cruce entre la psicología y la sociología. Desde una psicología social crítica de los cuestionamientos a la existencia de leyes universales que pueden explicar el comportamiento social y un interés por explorar las instituciones y prácticas que promueven la desigualdad, desde la asignatura se procura realizar un recorrido por los alcances, las limitaciones y los entrecruzamientos de las principales teorías y métodos de la psicología social en la actualidad, y sus debates contemporáneos a nivel local e internacional. Particularmente, en esta materia se dedica una de las unidades a la formación de prejuicios, estereotipos y discriminaciones, más específicamente: estereotipos de género, xenofobia, homofobia y se resalta el aporte de la teoría de género al análisis del sexismo ambivalente. Y una segunda unidad, a la agresión y la violencia, y se menciona: nociones de agresión y violencia; manifestaciones de la agresividad; y violencia en el contexto social: el caso de los medios de comunicación y la violencia machista.

En Epistemología de las Ciencias Sociales, las reflexiones en torno a la producción del conocimiento científico recorren los distintos periodos, escuelas y disciplinas que pretendieron hegemonizar el campo de la epistemología. Particularmente se menciona que a partir de los años ochenta, se fue desplegando la epistemología feminista y las corrientes ligadas a la deconstrucción como el postestructuralismo y el postfundacionalismo, que renovaron las interrogaciones sobre la práctica científica, la filosofía y las ciencias sociales y sus procedimientos de verdad, objetividad, fundamentación y conocimiento. En este programa se despliega una unidad en particular, "Epistemología y Deconstrucción” destinada a los estudios 
de género como enfoque para el análisis epistemológico: deconstrucción y diferencia. Logocentrismo y falocentrismo. Epistemología y feminismos. Género y sexo, identidad y performatividad.

A partir de la lectura comprensiva de la fundamentación, los objetivos y contenidos de cada programa se pueden destacar dos puntos: en primer lugar, las asignaturas vinculadas al área "historia social" tienden a la incorporación de estas temáticas a través de la inclusión de "las mujeres" y su participación en los distintos periodos históricos. En esta área se politiza la experiencia de las mujeres, se reconoce su lugar y sus aportes como fuerza política. En tanto que desde la psicología social se hace énfasis en los condicionamientos en la construcción de la subjetividad, los cuerpos y los deseos. Y desde la epistemología se recupera la epistemología feminista como una corriente que renovó las interrogantes sobre la práctica científica y habilitó nuevas preguntas que hasta el momento estaban invisibilizadas.

En segundo lugar y vinculado con la organización curricular de los contenidos, se pueden reconocer tres formas en su estructuración: a) como contenidos específicos que se abordan en unidades específicas; b) como una perspectiva que permea el enfoque de la asignatura; y c) como una combinación entre ambas, formando parte del enfoque y a su vez, abordándose en unidades específicas.

En los casos en que se abordan como "contenidos específicos", su mención se refiere a contenidos a tratarse a largo de la cursada, formando parte de unidades más amplias o de unidades específicas. En estos programas, los contenidos tienden a emerger como diferenciados del resto, pudiendo tener vinculación con las otras unidades o bien, permanecer sin conexiones como una temática que comienza y termina en sí misma. En esos casos, "el contenido" o "la unidad" puede configurarse en la atomización de la temática. Y en los casos que refieren al enfoque de la asignatura, su mención se explicita en la propia fundamentación, dando cuenta que estas problemáticas y perspectivas son inherentes a los fenómenos y procesos sociales y por lo tanto, requieren para su abordaje de los estudios de género.

\section{¿A quién se lee en la carrera de Sociología y qué lugares ocupan? Que nos dice la bibliografía de cada programa, además de los textos a leer}

En los apartados anteriores, me dediqué a analizar las presencias y ausencias de las problemáticas y perspectivas de géneros en los programas, como así también de la organización curricular de estos contenidos al interior de cada asignatura. En este apartado, analizo la bibliografía relevada en los 37 programas del bloque obligatorio. 
Para este relevamiento, plantee dos niveles de análisis: por un lado, de la cantidad y de las identidades sexo-genéricas de lxs autorxs de los textos disciplinares de la bibliografía obligatoria, optativa y recomendada; y por otro, de la cantidad y de las identidades sexogenéricas de lxs autorxs de los textos específicos del campo de géneros y sexualidades (de la educación sexual integral) en la bibliografía obligatoria, optativa y recomendada ${ }^{\text {viii }}$.

En relación al primer nivel de análisis, "cantidad e identidades sexo-genéricas de lxsautorxs de los textos disciplinares", organicé dos grupos: el primero, referido a la bibliografía obligatoria, y el segundo, a la bibliografía optativa o recomendada. A su vez, al interior de cada grupo, organicé cuatro subgrupos: 1) cantidad de textos disciplinares; 2) cantidad de autorxs de textos disciplinares; 3) cantidad de textos escritos por hombres; 4) cantidad de textos escritos por mujeres.

En relación a la bibliografía obligatoria, tienen 1489 textos disciplinares, con un total de 1728 autorxs. Del total de 1728autorxs, hay 1436 autores y 292 autoras. En este caso, del total de autorxs que escriben los textos disciplinares de la bibliografía obligatoria, el $83 \%$ corresponde a producciones de hombres y el 17\% a mujeres. En el recuadro que está a continuación, se pueden ver con claridad los cuatro subgrupos:

Tabla 2

\begin{tabular}{l|l|l|l}
\hline $\begin{array}{l}\text { Cantidad de } \\
\text { textos } \\
\text { disciplinares }\end{array}$ & $\begin{array}{l}\text { Cantidad de } \\
\text { autorxs }\end{array}$ & Cantidad de autores & Cantidad de autoras \\
\hline 1489 & 1728 & 1436 & 292 \\
\hline & & & \\
\hline
\end{tabular}

Fuente: elaboración propia.

En la bibliografía optativa, tienen 1127 textos disciplinares, con un total de 1322 autorxs. Del total de 1322autorxs, hay 1078 autores y 244 autoras. En este caso, del total de autorxs que escriben los textos disciplinares de la bibliografía optativa, el $81 \%$ corresponde a producciones de hombres y el 19\% a mujeres. En el recuadro que está a continuación, se pueden ver con claridad los cuatro subgrupos:

Tabla 3

\begin{tabular}{l|l|l|l}
\hline $\begin{array}{l}\text { Cantidad de } \\
\text { textos } \\
\text { disciplinares }\end{array}$ & $\begin{array}{l}\text { Cantidad de } \\
\text { autorxs }\end{array}$ & Cantidad de autores & Cantidad de autoras \\
\hline 1127 & 1322 & 1078 & 244 \\
\hline & & & \\
\hline
\end{tabular}

Fuente: elaboración propia. 
En relación al primer nivel de análisis, sobre la cantidad y la identidad sexo-genérica de lxsautorxs de los textos disciplinares, hay cinco puntos a destacar:

- En primer lugar, se puede observar que en la mayoría de los programas, ya sea, en la bibliografía obligatoria como optativa o recomendada, las mujeres ocupan el 17\% y 19\% de la autoría en los textos disciplinares.

- En segundo lugar, las asignaturas que presentan una mayor cantidad de textos escritos por mujeres son las pertenecientes al eje metodológico, más específicamente las materias de Metodología de la Investigación. En estas asignaturas, es notable el cambio en los porcentajes de autoría que asciende a un $35 \%$ y $50 \%$ ix.

- En tercer lugar, los textos escritos por mujeres que se utilizan en las asignaturas de Metodología de la Investigación son -mayoritariamente- producciones de las propias docentes de la cátedra, entre ellas, las Profesoras Titulares, Asociadas, Adjuntas y también las Jefas y Ayudantes de Prácticos.

- En cuarto lugar, al igual que lo que ocurre en las asignaturas de Metodología de la Investigación -que hay una gran cantidad de artículos escritos por las docentes de las cátedras-, en el resto de las materias analizadas ocurre algo similar. Los textos que se proponen en la bibliografía suelen ser escritos por las docentes de las cátedras. Esto significa que el $17 \%$ y $19 \%$ de los textos son producidos -en buena medida- por las docentes.

- En quinto lugar, una particularidad que presentan los textos producidos por mujeres es que suelen aludir a materiales introductorios, de apoyo o de entrecruzamiento de distintas teorías o autores. Y a su vez, estas producciones suelen publicarse como materiales o cuadernos de cátedra: "La Sociología de...", "La teoría social de...," "La política en...", "Introducción a la obra de...", siendo producciones que hacen alusión en todos los casos a teorías o conceptos desarrollados por hombres, y que en su mayoría suelen ser los llamados padres fundadores de la Sociología (Karl Marx, Max Weber y Emile Durkheim), como así también Antony Giddens, Baruch Spinoza, entre otros. Esta particularidad no se encuentra con tanto énfasis en las asignaturas de Metodología de la Investigación.

En torno al primer nivel de análisis, se puede observar que las mujeres tienden a ocupar un escaso lugar en la bibliografía de los programas y que su presencia se concentra en las asignaturas relativas a la Metodología de la Investigación, que a su vez, son materias 
conformadas principalmente por docentes mujeres que son quienes escriben los artículos que forman parte de los programas. En muchas de estas producciones suelen escribir artículos introductorios, lo que no significa que los hombres no lo hagan, la diferencia radica en que las mujeres tienden a aparecer a través de estas escrituras. En el caso de los hombres, es una excepción. A partir de este primer relevamiento se producen algunos interrogantes: ¿por qué las mujeres ocupan un escaso lugar en la bibliografía (17\% y 19\%)? ¿por qué en las asignaturas de Metodología de la Investigación ascienden a un 35\% y 50\%? ¿por qué en las cátedras que están conformadas mayoritariamente por mujeres hay mayor presencia de textos escritos por mujeres? ¿qué ocurre en las cátedras que están conformadas principalmente por hombres o cuando ocupan los lugares de profesores?

En relación al segundo nivel de análisis, "sobre la cantidad y las identidades sexogenéricas de lxsautorxs de los textos específicos del campo de géneros y sexualidades en la bibliografía obligatoria, optativa o recomendada", hay cuatro puntos a destacar:

- En primer lugar, la cantidad de textos específicos sobre géneros y sexualidades en la bibliografía obligatoria es de 33 textos. Esta cantidad representa $0,02 \%$ del total de 1489 textos obligatorios. En relación a la bibliografía optativa, la cantidad de textos es de 8 . Esta cantidad representa el $0,007 \%$ del total de 1127 textos optativos o recomendados.

- En segundo lugar, los textos que abordan las cuestiones específicas de género y sexualidades, ya sea en la bibliografía obligatoria como optativa o recomendada son escritos por mujeres. Entre ellas se destacan Judith Butler, Donna Haraway, entre otras.

- En tercer lugar, las asignaturas que incorporan textos específicos son las 6 materias que mencionamos en los apartados anteriores: Historia Social Moderna y Contemporánea, Historia Social Latinoamericana, Historia Social Argentina, Psicología Social (dos cátedras) y Epistemología de las Ciencias Sociales. La materia que presenta la mayor cantidad de textos específicos, tanto en la bibliografía obligatoria como específica, es Psicología Social.

- En cuarto lugar, hay dos materias que incorporan en su bibliografía estas temáticas pero que no la mencionan en la fundamentación o en los contenidos: psicología social (cátedra C) y una de las asignaturas de Metodología de la Investigación, que propone investigaciones que abordan temáticas como adolescencias, estereotipos, salud sexual y reproductiva. 
Al indagar en el segundo nivel de análisis, sobre la cantidad y las identidades sexogenéricas de lxsautorxs de los textos específicos del campo de géneros y sexualidades, surgen algunos interrogantes: ¿por qué las problemáticas de géneros y sexualidades ocupan un lugar marginal en la cantidad total de textos disciplinares? ¿por qué la mayoría de los textos se leen en psicología social? ¿por qué las mujeres son quienes mayoritariamente se encargan de escribir sobre estas problemáticas? Si las mujeres tuviesen una mayor presencia en la bibliografía de los programas, ¿se contribuiría a una sociología sensible al género, a una sociología con perspectiva de género, a una sociología feminista?

¿Por qué las problemáticas de géneros y sexualidades y la perspectiva de género ocupan un lugar relegado en el plan de estudios de la licenciatura?

A lo largo de este trabajo, es posible reconocer que las problemáticas y perspectivas de géneros se incorporan en las asignaturas obligatorias del ciclo de la licenciatura a partir de distintas disciplinas como Historia, Psicología y Epistemología, que conforman las áreas de "historia social", "metodología" y "complementarias". En este recorrido, también se puede observar que las asignaturas que conforman el área de "teorías sociológicas" no incluyen en sus programas estas temáticas. A partir de esta indagación, se podría anticipar provisoriamente que el abordaje de estas problemáticas y perspectivas requiere de los ingresos, cruces y articulaciones con otras disciplinas. Siguiendo este hilo y recuperando el relevamiento de la bibliografía, podría pensarse que además de los diálogos interdisciplinarios, también resulta necesaria la revalorización de las producciones realizadas por mujeres, teniendo en consideración que los textos que se refieren a estas problemáticas son escritos mayoritariamente por ellas.

Como plantee al inicio de este escrito, el hecho de poder indagar en la organización curricular y en los programas que conforman dicha carrera, permite comprender el lugar que ocupan las problemáticas de géneros y sexualidades, como así también la perspectiva de género en la formación de sociologxs. Como afirma Goodson (2000) en todo proceso educativo hay una iniciación dentro de una tradición que es definida por los juicios y las prácticas de lxs llamadxs- eruditxs especialistas. Al recuperar esta idea acerca del curriculum y la tradición, es posible preguntarse acerca de la tradición en la cual se está iniciando el estudiantado. ¿En qué tradición generizada se está iniciando? ¿Las tradiciones están marcadas por los saberes disciplinares que se enseñan (curriculum explícito) y se dejan de enseñar (curriculum nulo)? ¿Las identidades y posiciones sexo-genéricas de lxs docentes (en las composiciones de las 
cátedras) se configuran en una referencia identitaria para lxs estudiantes? ¿Los textos que se ofrecen para leer amplían el repertorio cultural y operan como una fuente de legitimación? ¿Los aprendizajes implícitos, ligados al curriculum oculto, refuerzan y potencian dicha tradición o por el contrario, contribuyen a resistirla e interrumpirla?

A partir del artículo de Mancuso (s/f) es posible conocer el recorrido de la carrera y de los distintos planes de estudio que se aprobaron a lo largo de los años. Este trabajo permite el reconocimiento del carácter provisorio de los saberes, como así también de las disputas políticas que motorizan los distintos grupos para definir cuál es el saber o los saberes válidos, legítimos y oficiales (DE ALBA, 1996). En este marco, Lopes Louro (2012) se pregunta acerca de ese "cuerpo de conocimientos" que conforman el plan de estudios y que se presenta como verdadero, legítimo, estable y universal. ¿Es realmente tan estable y universal? Según Lopes Louro, se trata de "extrañar el curriculum" (to queer), de hacerlo "extraño" a nuestra vista, de un acto y movimiento de desconfianza y puesta en duda, tanto del conocimiento como de las formas por las que llegamos a conocer y a no conocer (desconocer) otras (2012).

Pienso que ese es el espíritu del queering del currículo: pasar los límites, atreverse, desconfiar de lo que está allí y mirar de modo propio lo que está incluido para poner en situación embarazosa lo que está en ese "cuerpo de conocimientos"; en fin, enfrentar las condiciones en que se da el conocimiento (LOPES LOURO, 2012, p. 115)

Si se toma el curriculum como un texto "generizado" y "sexualizado", el queering implica desconfiar de ese "cuerpo", de las condiciones en las que se da y se legitima como tal. Implica también revisar dentro de ese "cuerpo" los silencios y las ausencias epistémicas que devalúan en el mismo acto de la omisión, cierto conjunto de saberes (DA CUNHA, 2015).

Desde Argentina, investigadorxs como Gogna, Pecheny y Jones (2010) llevaron adelante una investigación sobre la enseñanza de géneros y sexualidades en universidades públicas. A partir de esta investigación plantearon que los principales obstáculos para la inclusión de los estudios de sexualidades en el curriculum universitario depende de las dinámicas inerciales del sistema de educación superior, el conservadurismo de la academia y la valoración de las problemáticas y perspectivas de géneros como algo trivial, extraño y de menor interés académico. En las carreras de ciencias sociales, la visión predominante privilegia la lucha de clases, las metodologías positivistas, en tanto que las metodologías cualitativas, la esfera privada y la microsociología ocupan un lugar relegado.

En Argentina y en Latinoamérica ven (estas problemáticas) como un síntoma de la despolitización y el corrimiento de los problemas verdaderamente políticos, hacia el estudio de los gays, de los negros... son algunos obstáculos porque sigue jugando la 
idea marxista de la lucha mayor contra la lucha menor (GOGNA, PECHENY y JONES, 2010, p. 179).

En concordancia con dicha investigación, Barrancos (2013) plantea al recuperar un informe de Wallerstein (1997) que a pesar de la renovación del conocimiento científico en las diversas disciplinas, los planes de estudios presentan un innegable anquilosamiento y una deuda pendiente: la introducción de la dimensión de género en la formación de grado universitaria. También menciona que dicha deuda no se condice con la investigación académica ya que en el último cuarto de siglo hubo un desarrollo notable de las investigaciones centradas en las problemáticas de géneros y sexualidades. La distancia entre la investigación y la formación todavía es un hiato a resolverse en las universidades.

Desde México, Güereca Torres (2016) ha dado cuenta de los modos en que se invisibilizaron los aportes del feminismo, como fuerza social y como fuerza intelectual en el campo de la sociología y las ciencias sociales. Esa invisibilización implicó un borramiento de sus luchas vindicativas y de sus contribuciones al campo teórico-conceptual de análisis sobre "lo social". En este proceso, reconoce tres modos de invisibilización del movimiento feminista en tanto fuerza intelectual en el campo de la sociología: la invisibilización de las pioneras por exclusión y opresión, la invisibilización académica y la llamada "revolución feminista de la sociología”. Estos modos se caracterizan por la exclusión de las mujeres en los orígenes de la disciplina, la invisibilización de sus aportes en el período de institucionalización de la sociología en las universidades y posteriormente por el encapsulamiento en áreas determinadas (como los "Estudios de la Mujer") configurándose "sociologías de mujeres, por mujeres y para mujeres”. Según la autora, y siguiendo los aportes deEichler (1997), estos procesos comparten -y parten desde- un sesgo androcéntrico, que se articula con insensibilidad de género, dicotomismo sexual, familiarismo y sobregeneralización de la experiencia de los hombres.

En este contexto, es posible afirmar lo que Fox Keller denunció a principios de los años noventa desde la epistemología feminista: la ciencia está generizada y es masculina (1991). Esta afirmación da cuenta de la constitución de un "sistema género-ciencias", en el cual se van articulando los sentidos acerca de lo que se denomina que es "la ciencia", la forma en la cual "se hace" y quienes son las personas (más aptas, autorizadas y legitimadas) "para hacerla" (BOURDIEU, 1994). En este sistema, se generan dicotomías: público o privado, masculino o femenino, objetivo o subjetivo, y se comienza a asociar lo público, con lo objetivo y lo masculino, en contraposición a lo privado, con lo subjetivo y lo femenino (MAFFIA, 2007). Estos pares dicotómicos se apoyan y se definen mutuamente, tanto por la asociación como por 
la exclusión: la ciencia viene a ser un hecho objetivo, por lo tanto masculino, y por consiguiente separada del mundo de las mujeres. Está diferenciación sexo-génerica en el campo académico contribuye a la reproducción de la exclusión de las mujeres y la deslegitimación de su autoridad científica (BOURDIEU, 1994) y epistémica (MAFFIA, 2007). Este proceso de discriminación, exclusión e invisibilización es definido por Güereca Torres (2017) como violencia epistémica de género y se refiere al conjunto de prácticas científicas, disciplinares y congnitivas que invisibilizan los aportes de determinados grupos y sujetxs sociales a la construcción, discusión y difusión del conocimiento científico. Abarca el menosprecio colonial y social sobre los saberes y las experiencias de grupos y sujetxs sociales que son invisibilizadxs por su condición de género, de orientación sexual, de etnia o de nacionalidad.

\section{Conclusiones}

En este trabajo se llevó adelante una indagación sobre el plan de estudios y los programas que conforman el ciclo obligatorio de la carrera de sociología, con el propósito de generar una aproximación crítica sobre la formación en géneros y sexualidades (educación sexual) que se ofrece a lxs estudiantes y posiblemente futurxs docentes. Como se mencionó al inicio, este abordaje presenta potencialidades y limitaciones. Por un lado, al centrarse en lo prescriptivo del plan queda por fuera lo que acontece en las aulas, sin embargo, luego de esta indagación se renuevan los interrogantes por conocer lo que allí ocurre, por relevar las formas en que la educación sexual es llevada a cabo, ¿de qué modo los contenidos mencionados en los programas se abordan en las clases? ¿cómo se transmiten y se negocian significados acerca de la sexualidad? ¿qué lugares ocupan lxs estudiantes y cómo circula su voz, su palabra, sus experiencias? En definitiva, resulta de interés conocer lo que efectivamente ocurre en las aulas universitarias de la carrera de sociología.

Por otro lado, este abordaje permitió indagar acerca de las áreas y asignaturas que incorporan estas problemáticas y perspectivas, como así también sobre las formas en que aparecen mencionadas y organizadas curricularmente. A partir de este trabajo fue posible advertir las presencias y ausencias de estas problemáticas en los 37 programas analizados. Luego del relevamiento, es posible afirmar que la educación sexual se aborda principalmente desde tres áreas: historia social, metodológica y complementaria, y dicho abordaje requiere de las articulaciones y los diálogos con otras disciplinas como historia, psicología y epistemología. En relación al canon que se ofrece como lectura, los textos específicos sobre el campo de la 
educación sexual tiende a ocupar un lugar marginal, al igual que las producciones realizadas por mujeres.

A lo largo de este artículo, se fueron desplegando interrogantes y reflexiones acerca de la formación que se brinda en la universidad. Tuvo la intención de aportar al campo sociológico y a la educación sexual integral, procurando generar los movimientos necesarios para seguir pensando qué aportes pueden realizarse entre la pedagogía, la sociología y la educación sexual integral. Seguramente, a partir de estos diálogos, será posible desentrañar los sentidos vinculados a estos campos, y al mismo tiempo, imaginar una formación universitaria que revalorice sujetos, saberes y experiencias subalternizadas.

\section{Referências}

BAEZ, Jesica. Políticas educativas, jóvenes y sexualidades en América Latina y el Caribe. Las luchas feministas enlaconstrucción de la agenda pública sobre laeducación sexual. En: Movimiento de mujeres y lucha feminista en América Latina y el Caribe, Buenos Aires: CLACSO, p. 73-121, 2016.

BAEZ, Jesica. ESI ¿Con todas las letras? Un recorrido por los planes de estudios de la formación de profesores/as de Letras en la Ciudad de Buenos Aires. Exlibris, Buenos Aires, $\mathrm{n}^{\circ}$ 9, p. 144-155. 2020.

BARRANCOS, Dora. Estudios de género y renovación de las ciencias sociales en Argentina. Asociación Argentina de Sociología. Revista Horizontes Sociológicos, vol. 1, nº 6, p. 219 232, 2013.

BARRANCOS, Dora. Géneros y sexualidades disidentes en la Argentina: de la agencia por derechos a la legislación positiva. Cuadernos de intercambio sobre Centroamérica y el Caribe, vol. 11, $\mathrm{N}^{\circ}$ 2, p. 17-46. 2014.

BLANCO, Rafael. Universidad íntima y sexualidades públicas. La gestión de la identidad en la experiencia estudiantil. Buenos Aires: Miño y Dávila, 2014.

BOURDIEU, Pierre. El campo científico. Redes: revista de estudios sociales de la ciencia, Buenos Aires, no. 1, p. 129-160, 1994.

DA CUNHA, Mónica. El curriculum como Speculum. En: BACH, A. M. (Coord.) Para una didáctica con perspectiva de género. Buenos Aires: Editorial UNSAM y Miño y Dávila, p. 153-209. 2015

DE ALBA, Alicia. Curriculum: crisis, mito y perspectivas. Buenos Aires: Miño y Dávila, 1996.

EISNER, Eisner. Theeducationalimagination. New York: Macmillan, 1985. 
FELITTI, Karina. Regulación de la natalidad en la historia argentina reciente (1960-1987). Tesis de doctorado, Facultad de Filosofía y Letras, Universidad de Buenos Aires. 2009.

FOX KELLER, Evelyn. Reflexiones sobre género y ciencia. Valencia: Alfons, 1991.

GOGNA, Mónica; PECHENY, Mario; JONES, Daniel. Enseñanza sobre género y sexualidad en universidades públicas en la Argentina. En: ORTIZ-ORTEGA, A; PECHENY, M. (Comp.) Enseñanza universitaria sobre género y sexualidades en Argentina, Chile, China, México y Sudáfrica. Buenos Aires. Teseo, 2010, p. 153-191.

GOODSON, Ivor. El cambio en el curriculum. Barcelona: Octaedro, 2000.

GÜERECA TORRES, Raquel. Violencia epistémica e individualización: tensiones y nudos para la igualdad de género en las IES. Reencuentro. Análisis de problemas universitarios, México, vol. 29, no 74, p. 11-32. 2017. Disponible en:

https://reencuentro.xoc.uam.mx/index.php/reencuentro/article/view/929.

GÜERECA TORRES, Raquel. Claves para una sociología feminista. En: BLAZQUEZ, N. \& CASTAÑEDA, P. (Coords.) Lecturas críticas en investigación feminista. México: UNAMCEIICH, 2016, p. 91-123.

JACKSON, Philip. La vida en las aulas. Madrid: Morata, 1968.

LAVIGNE, Luciana. Una etnografía sobre sexualidades, género y educación. La educación sexual integral en la Ciudad de Buenos Aires como política de gestión de la sexualidad juvenil. Tesis de Doctorado. Facultad de Filosofía y Letras. Universidad de Buenos Aires. 2016.

LOPES LOURO, Guacira. "Extrañar" el curriculum. En: SPADARO, M; FEMENÍAS, M. (Comp.) Enseñar filosofía, hoy. La Plata. EDULP. p. 109-120, 2012.

MAFFIA, Diana. Epistemología feminista: la subversión semiótica de las mujeres en la ciencia. Revista Venezolana de Estudios de la Mujer, Caracas, vol. 12, nº 28, 2007.

MANCUSO, Mara. La carrera de sociología de la UBA, su currícula a través de los años. 1958-2011. Disponible en:http://archivosociologia.sociales.uba.ar/wpcontent/uploads/sites/34/2015/10/06-Doc-PlanesEstSocio-MaraMANCUSO.pdf

MORGADE, Graciela. Contra el androcentrismo curricular y por una educación sexuada justa. Revista Internacional de Educación para la Justicia Social (RIEJS), UAM México, vol. 6, no 2, p. 49-62, 2017.

MORGADE, Graciela; ALONSO, Graciela. Educación, sexualidades, géneros. Tradiciones teóricas y experiencias disponibles en un campo en construcción. En: ALONSO, G;

MORGADE, G. (Comp.) Cuerpos y sexualidades en la escuela. De la "normalidad" a la disidencia. Buenos Aires. Paidós, p. 19-40, 2008. 
MORGADE, Graciela; FAINSOD, Paula. Convergencias y divergencias de sentido en los talleres de Educación Sexual Integral de la formación docente. Revista Instituto de Investigaciones en Ciencias de la Educación, Buenos Aires, no 38, p. 39-62, 2015.

MORGADE, Graciela; FAINSOD, Paula; BAEZ, Jesica; GROTZ, Eugenia. De omisiones, márgenes y demandas. Las universidades y su papel cardinal en la educación sexual con enfoque de género. En: ROJO P; JARDON, V. (Eds.) Los enfoques de género en las universidades. Universidad Nacional de Rosario, Grupo Montevideo, p. 67-95. 2018.

MORGADE, Graciela et al. Educación Sexual Integral con perspectiva de género. Rosario: Homo Sapiens. 2016.

PECHENY, Mario; PETRACCI, Mónica. Derechos humanos y sexualidad en la Argentina. Revista Horizontes Antropológicos, Porto Alegre, vol. 12, nº 26, 2006.

PETRACCI, Mónica; RAMOS, Silvina. La politica pública de salud y derechos sexuales reproductivos en la Argentina. Aportes para comprender su historia. Buenos Aires: CEDES, 2006.

SARDI, Valeria. A contrapelo. La enseñanza de la lengua y la literatura en el marco de la Educación Sexual Integral. La Plata: Editorial de la Facultad de Humanidades y Ciencias de la Educación de la UNLP. 2017

SACRISTAN, Gimeno. Comprender y transformar la enseñanza. Madrid: Morata, 1991.

Documentos legales

ARGENTINA. Ley de creación del Programa Nacional de Salud Sexual y Procreación Responsable. 30 de Octubre de 2002. Disponible en: http://servicios.infoleg.gob.ar/infolegInternet/anexos/75000-79999/79831/norma.htm

ARGENTINA. Ley de creación del Programa Nacional de Educación Sexual Integral. 4 de Octubre de 2006. Disponible en:

http://servicios.infoleg.gob.ar/infolegInternet/anexos/120000-124999/121222/norma.htm

\footnotetext{
iEn este trabajo se utilizará la flexión "x" en los casos en los que se designe a lxs sujetxs, como un modo entre otros de tensionar el binarismo masculino y femenino, como así también la preeminencia del género masculino en las formas del plural. En los casos que sea necesario, se mencionara a lxs sujetxs como "mujeres" o "varones", a partir de la lectura que se realiza sobre los cuerpos socialmente construidos y re-producidos de manera constante. iiEl enfoque biomédico se focaliza en la anatomía y fisiología de la reproducción, colocando el énfasis en las "amenazas" y los "riesgos" que se corren por perseguir una vida sexual y genital placentera. Desde este enfoque la sexualidad es entendida desde la prevención. El enfoque moralizante se refiere a cuestiones vinculares y éticas. Se aborda la educación sexual desde sistemas normativos que señalan "lo correcto" y "lo incorrecto", y establecen cuales son las prácticas, los deseos y expectativas acordes a la edad y a la identidad sexo genérica. Desde este enfoque no se tienen en consideración los sentimientos y las experiencias reales de lxs estudiantes, la palabra de lxs adultxs se torna incuestionable en cuestiones de sexualidad.

iiiEn el 2017 se inició un proceso de revisión del plan de estudios, tanto de la licenciatura como del profesorado. Para profundizar: http://sociologia.sociales.uba.ar/presentacion-y-novedades/
} 
${ }^{i v}$ En esa misma época, se crearon las carreras de Economía (que funcionaba en la Facultad de Ciencias Económicas) y de Ciencias Antropológicas, Ciencias de la Educación y Psicología (que funcionaban junto con Sociología en la Facultad de Filosofía y Letras).

${ }^{\vee}$ El Ciclo Básico Común (CBC) se creó en 1984, y en el caso de la carrera de Sociología, las asignaturas a cursar son: Introducción al conocimiento de la Sociedad y el Estado, Introducción al Pensamiento Científico, Sociología, Economía, Ciencia Política y Antropología.

${ }^{v i}$ Dentro de las 9 materias optativas pueden elegir entre aquellas correspondientes a "Teorías sociológicas" y/o "Sociologías Especiales". Para el año 2020, en el bloque de "Teorías sociológicas" podían optar entre un total de 26 materias, en tanto que, en el bloque de "Sociologías especiales" podían hacerlo entre 56 materias. La cantidad de asignaturas optativas puede variar a lo largo de los años en función de las nuevas materias que se proponen y se aprueban desde la carrera.

viiLos 37 programas que forman parte del objeto de estudio de este artículo, son aquellos que se encontraban disponibles en la página oficial de la carrera de sociología (UBA) durante el año 2020, periodo en el cual se llevó a cabo el trabajo de campo.

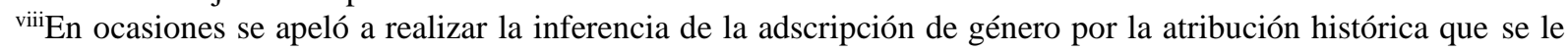
otorgó al nombre de pila. Esto resultó ser un obstáculo, pues aun cuando no partimos de supuestos binarios sexogénero no contamos con herramientas suficientes para poder distinguir los textos por otras categorías.

${ }^{\mathrm{ix}}$ Los porcentajes resultan del relevamiento de las tres asignaturas de Metodología de la Investigación (I, II y III) y de las tres cátedras que dictan dicho espacio curricular. 